\title{
L'ENSEIGNEMENT DU FRANÇAIS LANGUE-CULTURE : REPRÉSENTATIONS, ENJEUX ET CHOIX
}

\author{
Razika BENTAYEB
}

\author{
Université 8 Mai 45, Guelma, Algérie
}

bentayeb.razika@yahoo.fr

Article reçu le 16 novembre 2016 | révisé depuis le 19 novembre 2016 | accepté le 31 décembre 2016

\begin{abstract}
RÉSUMÉ. S'il est légitime que chaque langue-culture en tant que système ait ses défenseurs qui veillent à la préserver en gagnant plus d'espace par rapport à d'autres systèmes, la classe du français langue étrangère, dans le contexte algérien, se présente comme un des lieux de rencontre des langues-cultures, d'échanges mais aussi de tensions, voulu par les politiques comme un lieu de défense et de préservation. Sachant que l'institution éducative algérienne veille à la formation de la personnalité des apprenants en prenant en charge en premier lieu les valeurs identitaires qui se déploient à travers la notion d'algérianité; ainsi préserver la culture des apprenants c'est préserver l'algérianité de ces derniers. Mais, en quoi et comment peut-elle contribuer à la préservation des intérêts identitaires nationaux à travers l'enseignement d'une langue étrangère à savoir le FLE ? Les résultats de l'analyse du manuel scolaire de français de la 3ème AS ont démontré que l'institution éducative algérienne propose un enseignementapprentissage du français langue étrangère réduit de sa substance culturel alors que les didacticiens insistent plus que jamais sur le fait que l'on ne peut enseigner-apprendre une langue sans sa culture.
\end{abstract}

Mots-clés : FLE, l'écart, institution éducative, manuel scolaire, représentation.

\begin{abstract}
While it is legitimate that each language-culture as a system has its defender who are careful to preserve it by gaining more space compared to other systems, the class of French as a foreign language, in the Algerian context, presents itself as one of the meeting places of languages and cultures, exchanges but also tensions, wanted by politicians as a place of defense and preservation. Knowing that the Algerian educational institution is concerned with the formation of personality, which is the first component of the overall profile, learners in the first name in charge first of all of the identity values deployed through the notion of Algerianism; Thus preserving the culture of the learners is preserving the algerianity of the latter. However, what and how can it contribute to the preservation of national identity interests through the teaching of a foreign language, the FLE? The results of the analysis of the French textbook of the third ASE showed that the educational institution proposes a teaching-learning of the FLE reduced of its cultural substance whereas the didacticians insist that one can not teach- Learn a language without her culture.
\end{abstract}

Keywords : educational institution, FLE, gap, representation, textbook 


\section{INTRODUCTION}

« Les progrès vertigineux de la mondialisation, dans le commerce, le tourisme, les médias, la culture et à un degré moindre, l'école, font que toutes les cultures se trouvent soumises à une série de mélanges de diverses natures et doivent bien trouver leur chemin pour gérer cette abondance et cette mixité »(Cuq, 2003, p.137). La tentation de l'uniformisation par la puissance, l'argent et les médias a fait que nous vivons un affrontement interculturel où les dominants entreprennent à la réduction de la voix de l'Autre. Les pays, pour protéger leurs langues-cultures, se voient emprunter le chemin d'investir dans les espaces existants mais n'appartenant à aucune d'entre-elles, c'est investir dans «l'écart ».

Cet article vise à montrer comment l'institution éducative algérienne a investi dans l'écart comme moyen de préservation de l'algérianité qui constitue le noyau de la personnalité et de l'identité des apprenants, futurs citoyens. Ce qui a suscité notre intérêt est que tous les discours ainsi que les documents officiels de la politique et le système éducatif tels que la loi d'orientation, le référentiel des programmes, etc. insistent sur la dimension identitaire algérienne qui doit être ancrée tout au long de leur cursus scolaire.

Échapper à la tentation de l'uniformisation qui veut transformer la planète en un « village global » c'est préserver "l'écart ", car son inexistence conduira à l'inexistence de la pluralité linguistique et culturelle. C'est la différence, « l'écart » qui crée le mouvement, c'est elle qui permet l'enrichissement et la progression c'est elle aussi qui assure la continuité et la préservation. Elle donne à chaque entité une identité et une affirmation, chacune dans sa place. Mais cette distance, plus au moins importante entre une langue-culture et une autre est souvent un objet de conflit. Chacune veut se l'approprier pour garantir sa survie voire même son hégémonie.

Convaincus de l'existence d'une seule civilisation, celle qui est construite par l'Homme. L'Homme, à priori dans ce cas, est le même, il parle une langue et appartient à une culture, et le rêve d'un « village global » devient réalité. Cependant, « la malédiction de la tour de Babel » a fait qu'il y ait diversité et différence dont le mérite revient à « l'écart ». Mais, rappelons-le, l'homme a toujours cette volonté de dominer, d'être le meilleur, hanté par l'esprit de supériorité, de vouloir tout contrôler et s'imposer en imposant sa langue et sa culture sur l'Autre ; cet Autre « produit de l'écart », qui n'est que le reflet de lui-même mais qui, surtout, ne parle pas sa langue et ne partage pas sa culture.

Partant de l'idée qu'à partir de l'entredeux (l'écart) on peut analyser le mouvement de création, cheminement et progression de deux pôles, mais aussi on peut analyser les conflits qui s'accentuent davantage quand ces deux pôles, complètement différents linguistiquement et culturellement, coexistent sur le même espace géographique.

Pour certains : «L'inter » est la réalité première et fondamentale que personne ne nie ; quand deux systèmes coexistent ensemble, un espace d'échange se crée, et le plus fort le remportera et dominera. Comment, à ce moment, se protéger, contre l'Autre pour préserver ses valeurs et les promouvoir, se renforcer ? Comment se prémunir en préservant surtout «l'écart » qui donne " existence et valeur », maintenir cette distance au risque d'être envahi et absorbé ? A l'instar de beaucoup de pays, l'Algérie se présente comme un espace plurilingue dont deux importantes languescultures complètement distinctes, coexistent à savoir : l'arabe et le français.

Respectivement, la première est la langue officielle et nationale du pays, elle est aussi la langue de la scolarisation. La deuxième est une langue étrangère. En lui attribuant ce statut, nous l'insérant dans le rang d'inconnu, celui qui n'appartient pas à nous, qui nous est extérieur, different, distinct et éloigné.

Cependant, cette deuxième langue (FLE) a un fort ancrage dans le paysage social et économique du pays. Cette forte présence est due à la colonisation, 130 ans ou plus de présence linguistique et culturelle ne peut être effacée à coup de textes et de lois, encore moins si cette langue continue à véhiculer la science et la technologie. Elle est la langue de l'enseignement à l'université de 
la quasi-totalité des filières scientifiques et technologiques. Aussi, la réalité actuelle indique que la langue française est devenue un constituant de l'environnement linguistique et culturel algérien. Ainsi : sans être officielle, elle véhicule l'officialité, sans être la langue d'enseignement, elle reste une langue privilégié de transmission du savoir, sans être la langue de l'identité, elle continue de façonner de différentes manières et par plusieurs canaux l'imaginaire collectif, sans être la langue de l'universalité, elle demeure la langue de l'université. (Karima, 2007, $\mathrm{p}, 20)$

Vouloir préserver sa langue-culture, à assurer son existence d'abord et à la faire ensuite, propager en gagnant plus d'espace par rapport à d'autres systèmes est légitime et la classe de langue étrangère se présente à ce moment non pas comme un des lieux de rencontre des langues-cultures, d'échanges mais aussi de tensions, voulu par les politiques comme un lieu de défense et de préservation.

La classe du FLE dans le contexte algérien est l'un des lieux où la culture première de l'élève et la culture étrangère entrent en relation. Dans cette perspective, les outils d'enseignement-apprentissage de la langue étrangère, tel que le manuel qui est le support le plus utilisé par les élèves et les enseignants selon les enquêtes, représente un intérêt sociologique particulier : «Il met à plat la relation à l'étranger qu'une société donnée veut offrir en modèle à ceux qu'elle éduque ». (Zarate, 2004). La nouvelle loi d'orientation sur l'éducation, le référentiel général des programmes et le guide méthodologique d'élaboration des programmes, le confirment explicitement : «Il est impératif qu'il y ait une formation de la personnalité de l'élève aux valeurs de l'identité nationale qui se définie comme : identité historique collective, commune et unique » (Loi d'orientation sur l'éducation nationale, 2008, p.9). Ainsi que sa formation aux : «valeurs d'ouverture sur le monde et l'universel pour accepter l'Autre dans sa diversité culturelle et religieuse et idéologique et coopérer avec lui selon des intérêts librement partagés. » (Loucif, 2015, p.6)

En visant l'assimilation des principes de l'appartenance nationale et culturelle le manuel scolaire occuperait dans ce cas une position paradoxale; tel un cheval de Troie, il devrait promouvoir l'enseignementapprentissage d'une langue et donc d'une culture étrangère alors que le système, élaborateur du manuel, est orienté vers la promotion de l'identité et la culture nationale, à savoir l'Algérianité.

Comment le manuel scolaire de français de la 3ème année secondaire, comme support d'enseignement-apprentissage, peutil intégrer, à travers ses contenus, la problématique de l'identité et de l'Altérité ? Comment le concept de représentation, comme notion largement investie par l'institution politique et éducative qui veut l'ajuster à ses craintes, besoins et finalités, devient opératoire dans la conception du manuel, comme une façon d'avoir une mainmise pour maintenir « l'écart »?

\section{MÉTHODE}

Sachant que «Les images et les conceptions que les acteurs sociaux se font $\mathrm{d}^{\prime}$ une langue, de ce que sont ses normes, ses caractéristiques, son statut au regard d'autres langues, influencent largement les procédures et les stratégies qu'ils développent et mettent en œuvre pour apprendre cette langue et en user » (Moore, 2001, p.9). Cette attention porte sur les lieux physiques où ladite langue est parlée aussi bien que les locuteurs de cette langue. Elle dépasse ainsi la composante linguistique de la langue pour mettre en exergue le rôle des représentations de l'altérité. Ces dernières constituent une partie intégrante de la construction identitaire, de la relation entre soi et les autres et de la construction des connaissances. Les représentations permettent aux individus et aux groupes de s'auto-catégoriser et de préciser les caractéristiques qu'ils jugent pertinents pour construire leur identité par rapport à d'autres.

Elles sont ainsi à considérer comme une donnée intrinsèque que l'apprentissage qu'il convient d'intégrer dans les 
politiques linguistiques et des démarches éducatives. Ces démarches doivent pouvoir réconcilier des tensions a priori contradictoires entre un besoin d'autocentration et de rattachement au connu, et l'indispensable ouverture que nécessite l'apprentissage des langues. (Castelloti et Moore, 2002, p.21)

L'apprentissage d'une langue recouvre donc deux dimensions, la première relative à l'aspect linguistique et la deuxième se réfère au locuteur de la langue, à la construction de son identité et aux rapports que ce dernier développe avec d'autres usagers. Il va sans dire que, vu le caractère entremêlé de la langue et de la culture, ces deux dimensions s'enchevêtrent. L'image de l'autre est éminente dans le système humain de pensée puisqu'elle influence la construction de l'identité d'où son importance dans tous processus d'apprentissage.

Les représentations culturelles, relatives aux langues d'apprentissage, font l'objet de plusieurs recherches. Ces représentations portent, non seulement sur les locuteurs d'une langue mais également sur les pays où se parle ladite langue (Castellotti et Moore, 2002) ; (Zarate, 2004).

Des études effectuées en suisse (Moore, 2001) ont abouti à des résultats pertinents tel que, les représentations que se font les apprenants d'un peuple ou d'un pays et qui sont généralement en relation étroite avec des attitudes vis-à-vis de la langue d'apprentissage et au degré de complexité de son apprentissage. Si les apprenants développent des représentations plutôt négatives d'un pays, il en résulte souvent une image négative, fortement stéréotypée, de la langue en question.

Certains auteurs irons même jusqu'à établir «une relation entre les jugements de difficultés et de réussite dans l'apprentissage et la valorisation du pays concerné » (Castellotti et Moore, 2002, p.12). Dans ce sens, quelles représentations se font les apprenants sur la France et par ricochet sur la langue française, ou plutôt quelles représentations veut-elle l'institution éducative qu'ils se fassent? Pour répondre à cette question, nous nous sommes appuyées sur une méthode descriptive analytique entre quelques textes et illustrations pris dans
Ministère de l'éducation nationale (2008). La finalité étant de répondre à la problématique posée.

Nous avons pris du manuel scolaire de français langue étrangère, constitué de quatre projets pédagogiques, de chaque séquence un texte ainsi que les illustrations qui y figurent. Nous avons procédé à leurs descriptions et à l'analyse de la présence des éléments renvoyant à la France et à la culture française.

\section{RÉSULTATS ET DISCUSSION Résultats \\ Textes et illustrations pris du manuel de français}

Nous avons pris un échantillon de 8 textes sur une cinquantaine dont la moitié sont écrits par des auteurs algériens tel que l'historien Mahfoud Kaddache. Les autres textes appartiennent à des auteurs étrangers (Français et autres) dont lesquels ils traitent des sujets relatifs à la santé, la protection de l'environnement, l'enseignement dans le monde, les OGM, l'informatique, les recherches en médecine, etc. à côté d'autres textes appartenant à des écrivains de nationalité différente tels que: Gogol, P.M Orlan, A. Makine, A Baricco, Guy de Maupassant, et T. Gautier.

Texte $n^{\circ}$ 1: «La population urbaine en Algérie dans les années 1920 » (Kaddache, 1981 cité dans Ministère de l'éducation nationale, 2008, p.20)

Une première lecture du titre nous montre que l'auteur, l'historien algérien Mahfoud Kaddache, donne des statistiques sur les populations d'Algérie dans les années 1920. Cette population était constituée de musulmans, d'indigènes ainsi que des européens, et comment la politique française a fait en sorte de naturaliser les français et les européens pour que le taux d'accroissement de la population de ces derniers soit supérieur par rapport à celui des musulmans qui selon l'auteur restaient « cantonnés dans leur bled ». La raison de cette politique est que les français avaient peur et craignaient de voir les masses rurales constituer une force révolutionnaire dangereuse. Ce texte met en lumière la politique du colonisateur, 
il ne fait aucune référence ni à la culture française, ni à la culture occidentale, par contre il y a quelques références à la culture algérienne telle l'utilisation du mot « Bled »

Texte $n^{\circ} 2$ : «Le $1^{\text {er }}$ novembre 1954 à Khanchela" (Boubakeur, 1976, cité dans Ministère de l'éducation nationale, 2008, p.33)

L'auteur Salem Boubakeur, qui est un algérien, relate un fait historique le jour où Abbès Laghrour rentra de Batna, il s'agit d'un témoignage d'une personne qui a participé au fait raconté. L'auteur évoque la plate-forme politique du FLN, la description du drapeau algérien «(..) les deux tracts avaient en en-tête deux petits drapeaux vert et blanc entrecroisés et frappés du croissant et de l'étoile rouge ».

L'auteur décrit comment Laghrour Abbès à la suite de la lecture de ces tracts disait sans cesse: «Allah akbour le grand jour est arrivé », l'auteur évoque les mots de passe pour les opérations du déclenchement de la guerre "Khaled et Okba » deux noms qui sont, à notre avis, symboliques: le premier renvoi au célèbre guerrier Arabe Khaled Benoualid et le deuxième à Okba Ben Nafaa, le célèbre conquérant Arabe.

Nous remarquons que dans ce texte, il n'y a pratiquement aucune référence à la culture française. Au contraire, il y a beaucoup de référence géographique, religieuse et militaire renvoyant à l'Algérie telles que Batna, Benboulaid, Chihani, FLN, drapeau vert et rouge, croissant et étoile, allah akbeur, 1e novembre 1954, Khaled, okba, etc.

Texte $n^{\circ} 3$ : Poème « Chant populaire kabyle» (Benbrahim, s.d. cité dans Ministère de l'éducation nationale, 2008, p.24)

L'auteur dans ce poème relate la tragédie qu'Alger et la Kabylie ont été dévastées par l'armée française, et que cette dernière leur a demandé de se rallier à son armée et comment les habitants de ces deux régions ont refusé.

Dans ce poème, il existe peu de références à la culture française «képi, maréchal ", au contraire, l'auteur utilisait des références renvoyant à notre culture tels que : des références religieuses « le jour de l'aïd, rompre le jeune », des références sociales « la tribu de ait Iraten » des références régionales «icherriden, Kabylie, Alger » des références historiques « la célèbre guerrière lala fathma sœur de Sidi Tahar»

Texte $n^{\circ} 4:$ "Faut-il dire la vérité au malade? "(Viansson-Ponte et Schwartzenberg, 1997 cité dans Ministère de l'éducation nationale, 2008 p. 80)

Dans ce texte, qui est un débat d'idée, écrit par P.Viansson-Ponte, journaliste français et éditorialiste au Monde, et L. Schwartzenberg, cancérologue français, chacun des deux essayait de convaincre l'autre en défendant sa thèse par des arguments qui lui semble les plus efficaces pour atteindre son but.

Le premier essaie d'argumenter en faveur de sa thèse qui selon lui «Il ne faut pas désespérer le malade », c'est-à-dire que l'auteur est contre le fait de dire la vérité au cancéreux qui va mourir. En défendant sa thèse, le journaliste a utilisé des arguments tels que : "l'homme est le seul être vivant qui sache qu'il doit mourir, pourquoi donner à sa vie la compagnie quotidienne de la mort? At-on le droit de le désespérer? S'il guérit à quoi sert de lui dire ce qu'il avait puisque, il va continuer à vivre avec la hantise de la rechute. On lui impose l'angoisse, un désespoir parfaitement inutile ».

L'antithèse est annoncée à travers l'articulateur d'opposition «pourtant», pour le cancérologue, «le simple respect de l'homme exige qu'on lui dise ce qu'il en est. Le médecin ne doit pas mentir » "Ce qui est terrible quand on ment au malade, ce n'est pas de lui dissimuler la vérité, c'est de lui cacher ce que les autres savent... une telle situation va créer des relations entièrement faussées où se mêlent la pitié et l'hypocrisie. »

Deux noms français renvoyant respectivement au journaliste du journal le Monde P.Viansson-Ponte et au cancérologue L. Schwartzenberg apparaissent dans ce texte. Ce dernier contient des valeurs humaines et universelles telles que: dire la vérité, ne pas imposer l'angoisse et le 
désespoir aux malades, être solidaire avec les cancéreux, etc.

Texte $n^{\circ} 5$ (Rousseau, 1994, cité dans Ministère de l'éducation nationale, 2008, p. 93)

C'est une lettre rédigée par l'écrivain, philosophe et musicien Genevois francophone Jean Jacques Rousseau, et extraite de son livre rêveries d'un promeneur solitaire. Cette lettre est destinée à Madame de Francueil de son vrai nom Marie -Aurore de Saxe, comtesse de Horn, écrite à Paris le 20 avril 1751.

Dans cette lettre J.J. Rousseau admet que les arguments de son interlocutrice sont recevables pour mieux leur opposer des arguments contraires. D'ailleurs il a commencé sa lettre par : «Oui, madame, j’ai mis mes enfants aux enfants-trouvés (organisme qui correspond aujourd'hui à l'assistance publique) ». Rousseau admet d'avoir abandonné ses enfants et d'avoir chargé de leur éducation l'établissement qui est fait pour cela. Il défend sa thèse en avançant des arguments tels qu'il « ne pense pas les nourrir, qu'il veut être tranquille d'esprit pour faire son travail qui est purement lucratif. Il préfère que ses enfants soient orphelins que d'avoir pour père un fripon ". Nous pouvons dire que ce texte contient des éléments renvoyant à la culture française :

- Références littéraires: J.J. Rousseau, Madame de Francueil qui est une Comtesse. Ce denier est un titre de noblesse dont l'origine remonte aux premiers empereurs romain. C'est aussi l'un des plus élevé de la hiérarchie nobiliaire européenne.

- Des lieux : Paris, Enfants-Trouvés.

Texte $n^{\circ} 6$ "Appel au Peuple Algérien" (Front de libération nationale, 1954 cité dans Ministère de l'éducation nationale, 2008, p.128)

Il s'agit d'un texte exhortatif extrait du premier appel du 1e Novembre 1954, adressé par le Secrétariat Général du FLN au peuple algérien. Ce texte vise à faire réagir en interpellant le peuple algérien qu'il doit se mobiliser et agir pour le bien-fondé des vues du FLN dont le but demeure l'indépendance Nationale dans le cadre Nord-Africain. Dans cet appel, nous constatons qu'il n'y a que de références historiques et politiques renvoyant à l'Algérie telles que: «FLN, 1 novembre 1954, patrie, cause nationale, nord-africain, etc.»

Texte $n^{\circ 7}$ «Appel de l'Abbé Pierre du 1 février 1954 sur radio de Luxembourg" (L'abbé PIERRE, 1954, cité dans Ministère de l'éducation nationale, 2008 p.139)

L'auteur de ce texte, pour convaincre ses interlocuteurs et les pousser à réagir contre les gens qui meurent gelés à cause du froid et parce qu'ils n'ont pas où se loger, choisit des arguments qui font appel à la raison et aux sentiments des auditeurs pour les sensibiliser: «je vous prie, aimonsnous ", " chacun de nous peut venir en aide aux «sans-abri » il nous faut pour ce soir : 500 couvertures... » «Devant leurs frères mourant de misère, une seule opinion doit exister entre hommes: la volonté de rendre impossible que cela dure ». Nous remarquons que ce texte est riche d'éléments de la culture française :

- Noms de lieux: boulevard Sébastopol, Panthéon, rue de la montagne sainte Geneviève, Courbevoie, France, Paris, Hôtel Rochester, rue de la Boétie.

- Eléments divers: tentes américaines, radio de Luxembourg, Soupe, Poêles catalytiques.

- Eléments religieux: sainte Geneviève, l’Abbé Pierre.

Texte $n^{\circ} 8$ : «La main» (Guy de Maupassant, 1883, cité dans Ministère de l'éducation nationale, 2008 p.182)

Dans cette nouvelle, Guy de Maupassant, écrivain français, évoque l'une des coutumes de l'île française de Corse, qui est la vendetta. Cette dernière est une pratique ancestrale observée par une famille qui organise sa vengeance contre une ou plusieurs personnes ayant attenté à la vie de l'un de ses membres.

Les apprenants peuvent, à travers, ce texte prendre connaissance de la vendetta, de 
l'écrivain Guy de Maupassant ainsi que l'ile française : La Corse

\section{Illustrations}

A travers des illustrations et photos observées dans le manuel, nous constatons qu'il y en a plus de références géographiques, littéraires et historiques de l'Algérie que celles faisant référence au monde extérieur, à l'autre. Cela peut être vu dans les images qui représentent entre autres: (1) des femmes algériennes portant des plateaux sur leurs têtes pour ravitailler les djounouds de l'ALN (p.53); (2) un regroupement d'hommes portant des bernous et des cheches, dans une région du sud algérien. Les palmiers et la nature du sol le démontrent (p.42); (3) un buste d'un roi ou d'un guerrier numidien (p.25); (4) une femme algérienne dans les camps (p.35) ; (6) des constructions traditionnelles algériennes (p.30); (7) un célèbre écrivain algérien d'expression française Kateb Yacine (p.40) ; (8) un monument se trouvant à Alger et construit en hommage aux martyrs, le célèbre "Makam el Chahid» (p.57); (9) une caricature du journal El Watan représentant trois squelettes portant des habits traditionnels algériens et s'appuyant sur des cannes en disant: "Nous sommes les victimes des bienfaits de la colonisation! » (p.99).

À part ces images, nous pouvons remarquer qu'il y a une illustration qui fait référence à la protection de la nature : deux mains tenant le globe pour signifier que l'homme doit protéger la terre (p.136). Il y a également une illustration qui représente la solidarité : deux mains serrées l'une contre l'autre (p.141).

\section{Discussion}

Les constats faits après cette brève analyse du manuel scolaire de français de la 3ème année secondaire illustre parfaitement ce qui précède :

- En effet, le premier projet pédagogique porte sur le texte historique, ce dernier met l'accent sur le caractère colonial et barbare du pays (France).

- Une forte présence des textes historiques traitant majoritairement de la colonisation française. Ainsi ils visent plus la préservation de certaines valeurs nationales et identitaires que l'ouverture sur l'Autre et de ce fait ils n'offrent aucun accès à une formation à l'altérité.

- Qu'il n'y a pas une réelle ouverture et accès à la culture française à part quelques références aux valeurs universelles en relation avec la solidarité, la protection de la nature, etc.

- L'insertion d'une illustration reflétant une scène purement algérienne dans un texte écrit par un auteur français (p 99), nous pousse à se demander si la langue française pourrait véhiculer la culture algérienne? pourrait-elle représenter la personnalité et l'identité algérienne?

A partir de ce qui précède nous pouvons dire que dans celui de français il existe un certain nombre de référence à la France, à son histoire, à sa géographie, à sa politique, à sa culture par l'intermédiaire des noms de personnages connus et qui représentent la culture française. Cependant, l'existence d'éléments faisant référence à la culture algérienne, à son histoire, à sa géographie, à sa politique sont à priori plus importants et présentent même une nette prédominance.

Les concepteurs du manuel de langue mettent en scène la culture de l'Autre mais aussi la leur, ce qui intensifie la complexité des représentations contenues dans ceux-ci. (Bougerra, 2008, p.23) explique «Le statut des langues étrangères et la conception de la culture qui en découle, les représentations, l'imaginaire enthnosocioculturel surdéterminent en grande partie les contenus socioculturels actualisés dans les manuels scolaires ». «L'à travers » la culture algérienne, on regarde la culture de l'Autre en faisant fi de l'entre-deux.

Cependant, est-il possible de favoriser un apprentissage réussi et efficace d'une langue- culture étrangère, en préservant l'identité et la culture d'origine ? Par souci de maintenir la distance au risque d'être envahie, quels seraient, dans ce cas, les mécanismes qui permettraient un apprentissage d'une langue étrangère en dehors de ces paramètres culturels ?

Certes, ça a été démontré actuellement que toute intervention pédagogique devra 
tenter de réconcilier le cognitif avec sa base affective. Ainsi un échange permanent rend indissociables les aspects physiologique, émotionnel et cognitif d'un comportement. La force de ces liens explique l'incidence majeure qu'ont les émotions sur les apprentissages. Une émotion perçue comme positive, associée à un apprentissage, facilitera la réussite, tandis qu'une émotion perçue comme négative pourra induire l'échec.

Un état émotionnel fort positif, un contexte remarquable, la motivation surtout intrinsèque et l'attention portée par l'individu à ce qui doit être mémorisé améliorent considérablement la mémorisation d'une information ou d'un apprentissage. La conception de ce dernier, avec l'avènement du constructivisme, se base sur la construction des connaissances. L'apprentissage est centré sur l'apprenant et repose sur l'évolution de connaissances antérieures, en fonction de l'expérience, des envies et des besoins de chacun.

Ces bouleversements théoriques donnent ainsi naissance aux pratiques dites actives ou expérientielles de l'apprentissage par l'action. L'on recherche une implication active de la part de l'apprenant qui interagit avec son environnement matériel et humain, en se fondant sur l'idée que l'action entraine un niveau d'intégration de l'information plus profond que la perception. En effet, dans l'action, il $y$ a nécessairement opérationnalisation, c'est-à-dire mise en application de concepts. L'apprenant doit non seulement acquérir des savoirs et des savoir-faire mais aussi pouvoir les opérationnaliser dans le cadre de situations concrètes. L'apprenant se retrouve donc actif et son apprentissage en est d'autant plus performant.

Le contexte dans lequel l'élève se sent interpellé totalement doit être enrichissant et chaleureux. Ainsi, les élèves d'une même classe qui suivent un même cours n'apprendront donc pas la même chose. Leurs représentations des notions abordées seront différentes car ils ne disposent pas à la base des mêmes acquis et ne partagent pas non plus le même style d'apprentissage. Ils garderont tous une trace de ce dernier, mais cette trace sera différente et spécifique pour chaque élève, de même pour leurs représentations.

Il conviendrait de ne pas ignorer ces différences et d'éviter de les combattre (consciemment ou inconsciemment). Au contraire, il faut intégrer dans les apprentissages de plus en plus la diversification des contenus, stratégies d'enseignement ainsi que des thèmes, en négociant ces derniers avec les élèves, en vue d'une plus grande personnalisation.

De plus, l'enseignant est investi d'un rôle majeur et est mandaté par la société pour éduquer et former les apprenants de langue à la pluralité linguistique et culturelle. Or, il semblerait que le discours des enseignants est loin d'être neutre. Ils s'est avéré que les enseignants, eux aussi, transmettent des jugements de valeur à l'égard de la langue, de ces locuteurs et du pays où se parle la langue.

De ce point de vue, ces enseignants risquent d'encourager les représentations stéréotypées qu'ont les apprenants, ce qui est à l'opposé d'une ouverture et d'une sensibilité à l'égard de l'altérité. D'autre part, il semble évident, que l'enseignant joue un rôle déterminant en tant que médiateur du contenu des manuels lors de l'interaction verbale en classe. Dans cette perspective, la nécessité d'une formation à la didactique du plurilinguisme s'avère indispensable.

Mais, ces éléments sont-ils suffisants pour apprendre une langue étrangère sans prendre en compte ces paramètres culturels? Certes, ces mécanismes peuvent aider à optimiser l'apprentissage du français. Seulement, dans le contexte algérien le passé historique, les enjeux politiques et éducatifs ainsi que le contexte national font que la relation entre la langue française et la culture française ne dépend plus des exigences des didacticiens s'accordant sur l'obligation que l'on ne peut « séparer langue et culture et que la seule maitrise des codes linguistiques n'est pas suffisante pour communiquer » (Barthelemy, Groux et Porcher, 2011, p.117). Mais, plutôt du postulat que la relation entre l'enseignement de la langue-culture est indissociable, n'est plus une évidence. 


\section{CONCLUSION}

Pourquoi vouloir décontextualiser le français? Le vider de sa substance culturelle? Cette peur de l'Autre est-elle justifiée? fondée? Il est naturel de se préserver contre l'Autre, maintenir l'écart, investir dans les représentations, renforcer l'algérianité. Pourtant l'on devient soi même que dans le contact avec les autres et l'on prend plus conscience tout à fois de sa spécificité et de sa pluralité. Que cette interaction entre l'autre et moi est nécessaire à la construction de l'identité : le moi a besoin de l'autre pour se construire.

Quand il y a différence, il y a écart, car l'écart c'est la différence. La préserver dans ce cas c'est préserver l'écart. Pourquoi déculturé le français si au contraire son enseignement me permet de mieux vivre ma singularité, et je ne suis moi-même qu'au contact de l'Autre, alors que l'identité profonde ainsi que la culture d'origine de l'apprenant sont conservées par le cerveau reptilien responsable de "réactions d'auto préservation et de repli défensif vers le connu, les racines, l'identité profonde» (Cuq et Gruca, 2005, p.108).

Réduire le français de sa substance culturelle ne fera qu'augmenter le risque de renfermement, de stagnation de l'esprit, de l'immobilité de la personnalité, son non épanouissement car c'est la rencontre, la différence qui m'affirme, me définit. L'image positive de l'Autre fait ressortir les défauts de soi. Ainsi l'Autre nous définit en tant qu'entité par un mécanisme d'opposition. L'identité se construit « l'à travers » l'Autre.

A travers le contenu du manuel scolaire de français de la 3ème A.S, notamment le texte historique, les acteurs du champ éducatif voudraient inciter les élèves à mettre des distances avec «la France » par le biais de «sa langue» de peur que les nouvelles générations oublient. Dans le cadre de l'algérianisation des programmes d'enseignement ainsi que les manuels scolaires, le français peut-il être algérianisé ? Cette approche méthodologique est-elle dictée par une approche idéologique sousjacente?

\section{REMERCIEMENTS}

Je tiens à remercier le comité éditorial de la revue Francisola pour la publication de cet article.

\section{RÉFÉRENCES}

Barthelemy, F., Groux, D., Porcher, L. (2011). Le français langue étrangère. Paris: L'Harmattan.

Bougerra, T. (2008). Approche écodidactique des représentations de l'interculturalité dans les manuels algériens de FLE enjeux et perspectives. Dans Kadi, L. (dir.). Cahiers de langue et de littérature, Manuels scolaires en classe de FLE et représentations culturelles (vol.5, p.1742). Mostaganem: Université Albelhamid ibn Badis Mostaganem.

Castelloti, V. et Moore, D. (2002). Représentations sociales des langues et enseignements. Strasbourg: Conseil de l'Europe.

Cuq, JP. (2003). Dictionnaire de didactique du FLE/FLS. Paris : CLE international.

Cuq, JP. et Gruca, I. (2005). Cours de didactique du français langue étrangère et seconde. Paris : CLE international.

Karima,

$$
\text { A.D. }
$$

Enseignement/apprentissage des langues en Algérie entre représentations identitaires et enjeux de la mondialisation. Synergie Algérie, 1, 173-180. Repéré à https://gerflint.fr/Base/Algerie1/karima .pdf

Loi d'orientation sur l'éducation nationale n08-04 (23 Janvier 2008).

Loucif, A. (2015, Novembre). Les programmes scolaires de deuxième génération fondés sur l'APC: cadre logique et modèle opératoire. Affiche présentée au colloque international «Approche par les compétences dans l'enseignement supérieur ». Skikda.

Ministère de l'éducation nationale. (2008). Manuel de français, Troisième année. Alger: Office National des Publications scolaires. 
Moore, D. (2001). Les représentations des langues et de leur apprentissage: Références, modèles, données et méthodes. Paris: Collection CREDIF-Essais, Didier.
Zarate, G. (2004). Représentations de l'étranger et didactique des langues. Paris : Didier Collection. 